\title{
Fanny Rubio oder der spanische Liebesdiskurs
}

Der literarische Text, mit dem wir uns in der Folge beschäftigen wollen, ist ebenfalls theoretischer und zugleich literaturwissenschaftlicher Natur, auch wenn sich seine Verfasserin nicht zuletzt als Romanautorin in Spanien und weit darüber hinaus einen Namen gemacht hat. Die Beschäftigung mit diesem Text soll uns ein erstes Mal mit den Spezifika des spanischen Liebesdiskurses vertraut machen, oder uns doch mit dieser Tradition enger in Berührung bringen. Die Verfasserin unseres Textes ist die Schriftstellerin Fanny Rubio, die in ihrem Hauptberuf als Professorin an der Universidad Complutense zu Madrid arbeitet. Ihr Buch hat den eher reißerischen Titel El embrujo de amar; und diese verhexte Liebe, die natürlich auch die Verzauberung, den encanto nicht ganz verloren hat, soll uns hier etwas näher beschäftigen. Es ist eine Arbeit über den spanischen Liebesdiskurs nach der Welle erotischer Literatur, die sich nach dem Tod von Generalísimo Franco und damit nach dem Ende der Diktatur über Spanien ergossen hat.

Der Band ist für uns im Rahmen unserer Vorlesung von sehr eigenem Interesse. Denn in gewisser Weise könnte man dieses Buch als eine spanische und zugleich als eine weibliche Replik auf Roland Barthes' Fragmente eines Diskurses der Liebe verstehen: freilich - das sei von Beginn an hinzugefügt - bei weitem nicht auf der Höhe der theoretischen Reflexionen des französischen Semiologen. Fanny Rubios Buch ist eher ein wenig im Plauderton gehalten, was aber den Vorzug hat, uns zu zeigen, wie Mann oder Frau heute in Spanien über das Thema Liebe sprechen kann. Ich darf sie also beruhigen: Die spanische Autorin hat kein Theoriebuch über die Liebe in den Spuren von Roland Barthes verfasst, sondern kokettiert nur ein klein wenig damit.

Ausgangspunkt für die gesamte Anlage des Bandes ist ein Treffen des Erzähler-Ichs mit Freundinnen und Freunden, darunter auch einigen ehemaligen Geliebten, bei einer madrilenischen Karaoke-Veranstaltung, wo die wunderschönsten Boleros mit Liebestexten gesungen und immer wieder in den Fortgang der Reflexionen der Ich-Erzählerin (alias Fanny Rubio) einbezogen werden. Wir sind bei der Lektüre also ganz tiefenentspannt, uns erwartet kein Traktat. Natürlich könnte man hier schon eine Grundidee vermuten, die uns immer wieder begleiten wird, die aber leider von Fanny Rubio nicht ausgeführt worden ist. Diese Grundfrage lautet: Wer spricht eigentlich, wenn wir von Liebe sprechen?

Bei Roland Barthes war das sujet amoureux ja in der Tat aus vielfältigen Fragmenten und Stimmen zusammengesetzt, die sich oftmals aus literarischen Intertexten herleiteten. Im Grunde ist es auch bei der Karaoke-Veranstaltung ganz genauso, denn auch hier werden die Subjekte im Grunde von den verschiedensten Fragmenten unterschiedlicher Herkunft gesprochen. Insofern bedient sich 
Fanny Rubio also desselben Strickmusters, nur dass ihre handelnden und liebenden Figuren geschlechtlich sehr wohl determiniert sind und vieles im Sinne von „Ella cantaba boleros“ - um Guillermo Cabrera Infante und seine Tres tristes tigres zu zitieren - vonstatten geht.

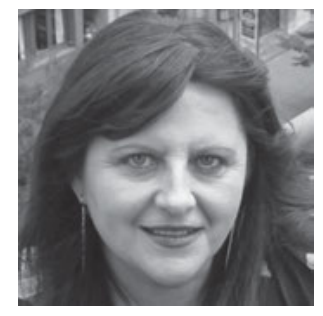

Abb. 60: Fanny Rubio, eigentlich Francisca Rubio Gámez (Linares, Jaén, 1949).

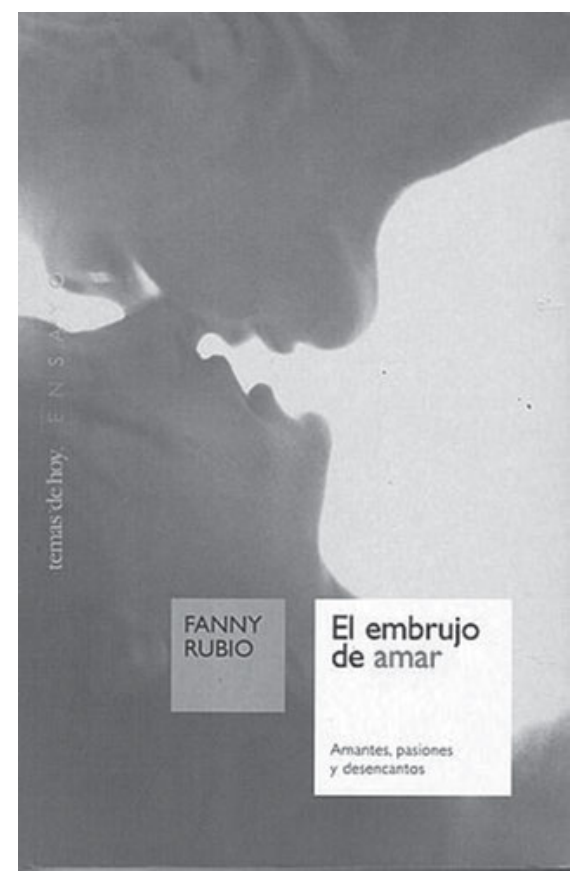

Abb. 61: Cover von Fanny Rubios El embrujo de amar.

Die nietzscheanische Frage „Wer spricht?“ ist in gewisser Weise im Liebesdiskurs also noch verschärft, denn wir folgen bestimmten Schemata, Versatzstücken und Formeln, die wir kulturell gelernt haben und die in uns sprechen und ihr EigenLeben führen. Erneut erkennen wir, dass diese Formeln von uns auch gefordert werden, wie dies bei der Formel „Ich liebe Dich“ recht anschaulich wird. Doch darauf werde ich noch mehrfach im weiteren Verlauf unserer Vorlesung zurückkommen. 
Gerade auch das Setting dieses Buches, sozusagen seine diegetische Anlage, mag verdeutlichen, wie wichtig die jeweiligen Orte des Sprechens für die Reflexion, und wie wichtig gerade auch die Verkörperungen für das eigene oder fremde Liebesgeschehen sind: wie also, um es mit anderen Worten zu sagen, der Liebesdiskurs buchstäblich vom liebenden Subjekt verkörpert wird. Denn entscheidend ist in dieser Veranstaltung die Einbeziehung des Liedtextes in das eigene Singen und damit in den eigenen Körper-Leib: All dies geht in die Realisierung, in die Darstellung und in die Identifikation mit der Liebe selbst im Sinne einer Inkorporation, einer liebevollen Einverleibung, ein. Der Liebesdiskurs will stets ein Diskurs mit Haut und Haaren und folglich ein leibhaftiger Diskurs sein.

Zugleich macht uns diese Szenerie natürlich auch auf eine zentrale Vermittlungsebene aufmerksam: nicht auf die hehre hohe Literatur und ihre intertextuellen Beziehungen, sondern auf die Alltagskultur und dabei vor allem auch die Liedtexte von Schnulzen oder die etwas anspruchsvolleren Texte von Boleros, die mit viel Sehnsucht und Herzschmerz gesungen werden. Das hört sich kunstvoller an, als es tatsächlich ist! Denn auch eine andere Literaturwissenschaftlerin, Iris Zavala, die viel über spanischsprachige Frauenliteratur gearbeitet hat, wird hier sowohl beim Singen als auch mit einigen Aufsätzen in den Band eingeblendet. Doch alles erfolgt eher auf der Ebene eines gewöhnlichen Plaudertons, einer beliebigen Konversation über Liebe in Spanien.

Der Ausgangspunkt des Bandes ist eine recht simple Frage - und so heißt auch das erste Kapitel: „Was wir sagen, wenn wir ,Liebe“ sagen“. Sie sehen, der Band stellt durchaus die elementaren Fragen nach der Liebe. Doch Fanny Rubio hütete sich freilich, eine klare Antwort zu geben. So ergibt sich ein zwangloser Spaziergang durch verschiedene Aspekte der Liebe unter besonderer Berücksichtigung der Geschlechterdifferenz, eine Unterscheidung, welche Roland Barthes gerade vermieden hatte. Es geht um die Liebe in Spanien zum Zeitpunkt der Veröffentlichung des Bandes, also mit einem gewissen Abstand zur Franco-Diktatur in einem demokratischen Land, in welchem freilich die Liebesdiskurse um die Jahrtausendwende je nach Sozialisation höchst unterschiedlich angelegt waren und wohl auch noch immer sind. Ich möchte Ihnen zunächst den Eröffnungssatz oder besser das incipit dieses Bandes kurz vorstellen, damit Sie einen direkten Texteindruck bekommen:

Es gibt einen Augenblick in unser aller Leben, in dem wir eine Liebeswahl vornehmen müssen, eine einzige, auf Grund derer wir die Konsequenzen über einen großen Teil unseres Lebens gut oder schlecht leben, selbst in jenen Ländern, in welchen man an eine Kultur der Berichtigung gewöhnt ist, mit ihrer jeweiligen affektiven, rechtlichen Entsprechung, der Scheidung, die selbst von jener Handvoll an Gläubigen aller Religionen anerkannt wird, die 
verstehen, dass die Tatsache, in einem bestimmten Alter eine bestimmte Wahl getroffen zu haben, nicht ohne weiteres und notwendig von einem Glücklichwerden gekrönt wird; oder aber zu einer lebenslänglichen Bestrafung all jener führen darf, die plötzlich entdecken, dass sie sich getäuscht haben. ${ }^{1}$

Fanny Rubios Band geht zum einen von Beginn an und nicht ganz zufällig vom Leben und damit von den Lebenserfahrungen eines jeden und einer jeden aus, um daran zunächst die These zu knüpfen, dass es in unser aller Leben sozusagen den Augenblick, den Moment einer Liebeswahl gebe, der für einen guten oder schlechten, aber in jedem Falle beträchtlichen Teil unseres weiteren Lebens und nicht nur unseres Liebeslebens - entscheidend sei. Dabei gibt es bei dieser Überlegung sofort einen Verweis auf das Scheidungsrecht, dessen historische Entwicklung im katholischen Spanien mühsam war. Denn selbst unter den Bedingungen einer späteren Scheidung, also der Auflösung der einmal gewählten Partnerschaft, sei die ursprüngliche Liebesentscheidung von großer Tragweite. So weit, so gut!

Mit dieser durchaus alltäglichen, vielleicht auch banalen Argumentation wird die Liebe mit ihren Partnerwahlentscheidungen in eine zentrale Stellung für das gesamte Leben gerückt. Offenkundig ist: Ein Wissen über die Liebe ist folglich ein Wissen über das Leben und zugleich auch ein Wissen, das es möglicherweise erleichtern könnte, das Leben in the long run zu meistern. Zugleich wird deutlich, dass der hier benutzte Begriff von Liebe ein sehr weitgefächerter, aber sicherlich nicht einfach romantischer ist, sondern vielmehr einer vernunftbetonten Partnerwahl gehorcht, da er eine Vielzahl von Konsequenzen - auch juristischer Natur - für das weitere Leben mitbedenkt. Wir sind hier vom ersten Satz an fast im Duktus eines Ratgebers für den Bereich der Liebe.

Fanny Rubios Text ist in gewisser Weise charakteristisch für eine literatura light, eine leichte - wenn auch hier nicht unbedingt schlüpfrige - Literatur, die keine wirklich fundamentalen Erörterungen im Schilde führt, sondern versucht, ein Thema auf eine eher gefällige Weise zu bearbeiten und dabei den Erwartungen des Lesepublikums weit entgegenzukommen. Denn diese Art von Literatur ist für eine breite Leserschaft kalkuliert, die überwiegend den Eingangsfragen zustimmend gegenübersteht. Ihr Kalkül rechnet von Anfang an mit einer substantiellen Verbindung zwischen Liebe und Lesen bei einer Leserschaft, die sich per se für das Thema Liebe interessiert. Damit aber gewinnt der Text wiederum an Interesse für eine eher kulturwissenschaftlich-soziologische Deutung.

1 Rubio, Fanny: El embrujo de amar. Amantes, pasiones y desencantos. Madrid: Temas de hoy 2001, S. 15. 
Darüber hinaus ist dieser Band auch in einer zweiten Hinsicht sehr charakteristisch, denn er spricht gerade die sexuelle Dimension der Liebe sehr direkt und wortreich, in zahlreichen umgangssprachlichen Varianten an. Dieses offene Gespräch, dieser offene Diskurs über die Liebe war in Spanien jahrzehntelang unter der Franco-Diktatur unterdrückt worden; und nach einer deutlichen Phase des destape, der ersten und auch kommerzialisierten Öffnung gerade auch für eine erotische Literatur, pegelte sich um die Jahrtausendwende eine gewisse Normalisierung des iberischen Liebesdiskurses ein, also des Sprechens über das zuvor verbarrikadierte und mit einer Vielzahl von Kautelen und Fangstricken versehene Thema.

Dies bedeutet mit anderen Worten, dass Sie als Studierende der Romanistik oder des Spanischen kaum einen besseren kommentierten Wortschatz über die Sprache der Liebe im Spanien des Übergangs zum 21. Jahrhundert finden können als dieses Buch von Fanny Rubio. Dabei nimmt die Verfasserin durchaus auf die alten Traditionen des iberischen und abendländischen Liebesdiskurses Rücksicht und baut sie in ihre Überlegungen mehr oder minder kunstvoll mit ein. So ist schon früh von Platons Gastmahl die Rede, auf das wir später noch zurückkommen werden, das uns aber bereits hier - wenn auch nur implizit - in der Figur des Alkibiades begegnet. Er tritt nämlich volltrunken in Platons Symposion auf und beklagt seine Liebe zu Sokrates, eine Liebe, die freilich unerwidert bleibt.

Doch die iberischen Traditionen bleiben im Vordergrund - und genau aus diesem Grunde beschäftigen wir uns ja auch mit diesem Buch, das uns ein wenig Einblick in den spanischen Liebesdiskurs und seine spezifischen Traditionslinien verschaffen soll. Ein zentraler Bezugstext ist für die Spanierin Rubio selbstverständlich in erster Linie Fernando de Rojas’ Celestina aus dem Jahre 1499, der in der Frühen Neuzeit die iberischen Diskurse der Liebe wesentlich mitprägte und eine Langzeitwirkung entfaltete. Denn in der Celestina begegnen wir für die iberische Welt zentralen Liebeselementen, wobei die schöne Melibea, die dank einer Kupplerin dem Calixto zugeführt wird, als eine wichtige Figur der Liebenden herauspräpariert und anhand der Verse „Yo soy la que gozo / Yo soy la que gano“ zu einer Art Urmutter der spanischen Frau wird, zu einer Frau, welche durchaus die Liebe genießt und die Lust an der Liebe bejaht.

Doch natürlich sind die Dinge nicht so einfach gestrickt - Denis de Rougemont lässt aus dem Bereich der Tragik von Liebesbeziehungen freundlich grüßen. Denn die Liebesfähigkeit der schönen Melibea ändert nichts an der Tatsache, dass sie nach dem Unfalltode ihres Calixto den Freitod wählt und sich vom Turm herabstürzt, eine psychoanalytisch besonders bedeutsame Handlung, die wir in der Symbolik von Sigmund Freuds Traumdeutung heute leicht zu entschlüsseln vermögen. Damit rückt der Liebesselbstmord im Rahmen tragisch verlaufender Liebesbeziehungen schon von Beginn der Frühen Neuzeit an in Spanien ein. Der 
Wille zum Leben erlischt, wenn der Wille zur Liebe seinen geliebten Gegenstand, sein Liebesobjekt, verliert.

Das weite Themenfeld des Liebesselbstmords spielt dann aber auch bei jenem Text eine Rolle, den schon Roland Barthes ins Zentrum seiner Überlegungen zum discours amoureux gestellt hatte, Johann Wolfgang Goethes Werther. Man könnte hier durchaus eine gewisse Parallele zwischen Barthes und Rubio erkennen, auch wenn die theoretische Durchdringung der Materie bei Rubio wesentlich geringer ist. Aber gerade in diesem theoretischen Anspruch unterscheidet sich der spanische vom französischen Band über die Liebe: Nehmen wir das Buch der spanischen Autorin also für das, was es leisten kann und uns in der Tat zu vermitteln vermag!

In einem großen Gegensatz steht freilich in beiden Büchern die jeweilige Behandlung der körperlichen Liebe. Hier macht sich besonders die jüngste spanische Geschichtserfahrung bemerkbar. In Fanny Rubios Band erkennen wir leicht noch immer die Spuren des destape in der nachfranquistischen Zeit, jene Jahre also, welche eine so starke Veränderung der Alltagskultur in Spanien gerade bezüglich des Liebesdiskurses wie der Liebespraktiken mit sich brachten. Dieser destape führte in der sogenannten movida zunächst zu einer liberación del porro, zu einer relativen Freigabe von Drogen, mit denen eine ganze junge Generation fast wahllos experimentierte, um dann selbstverständlich auch die tabuisierten Bereiche der Sexualität in Angriff zu nehmen. Spanien hatte unendlich viel aufzuholen: Eine ganze Generation sehnte sich nach einem anderen Leben und wollte rasch nachholen, was ihr zuvor ganz einfach vorenthalten wurde - oder besser: von dem sie glaubte, dass es ihr in all den langen Jahren der Diktatur schlicht entgangen sei. Die hektische, nach sofortigem Vollzug strebende Atmosphäre jener Jahre hat aus meiner Sicht vielleicht am besten der 1981 präsentierte Film von Carlos Saura mit dem Titel De prisa, de prisa eingefangen.

Spanien wurde zweifellos in jenen Jahren von einer ungeheuren Welle des erotismo erfasst, ${ }^{2}$ die bis heute im Grunde nicht völlig abgeebbt ist und eine Vielzahl von Ausdrucksformen gefunden hat, welche ebenso merkantiler wie auch ästhetischer Art sind. Gerade die Begründung von eigenen auflagestarken Reihen wie La sonrisa vertical hat das Thema von Sexualität, Erotik und Körperlichkeit zu einem so leicht in der Öffentlichkeit und in der Literatur behandelbaren gemacht, dass Fanny Rubios Buch in diesem Bereich sicherlich in keiner Weise tabubrechend wirkte, sondern der Erwartungshaltung eines spezi-

2 Eine schöne literaturwissenschaftliche Darstellung dieser Entwicklungen findet sich in Reinstädler, Janett: Stellungsspiele. Geschlechterkonzeptionen in der zeitgenössischen erotischen Prosa Spaniens (1978-1995). Berlin - New York: Schmidt 1996. 
fisch spanischen Lesepublikums vollauf entsprach. Man tut diesem Band ganz gewiss nicht Unrecht, wenn man ihn einem beherrschenden und nach Auflage schielenden mainstream zuordnet. Aber genau darin liegt auch seine Bedeutung für uns.

Am Ende des Textes steht - trotz aller Betonung der Gemeinsamkeit, des gemeinschaftlich verbrachten Karaoke-Abends und der vielen Erinnerungen an andere Menschen - die abgrundtiefe Einsamkeit. Es ist wahrlich kein Zufall, dass es gerade diese soledad ist, die den Schluss bildet und sich aller bemächtigt. Denn hierin drückt sich letztlich auch der tiefe Einfluss von Roland Barthes auf das Buch aus, in welchem er zwar bisweilen erwähnt wird, wo aber eine gewisse Scheu herrscht, mit seinen Verfahren $\mathrm{zu}$ arbeiten. Allzu übermächtig scheinen die Fragments d'un discours amoureux im Bereich des Schreibens über die Liebe vorzuherrschen. Und nicht umsonst hatte Roland Barthes die absolute Einsamkeit des liebenden Subjekts bei seinem Sprechen über die Liebe herausgehoben.

Was bei Barthes ganz am Anfang stand, steht nun bei Rubio also ganz am Ende: die Einsamkeit der Liebenden. Dort werden die Liebenden ganz schematisch den Geliebten gegenübergestellt, wobei letztere schlecht wegkommen, hätten sie sich doch opportunistisch und zugleich utilitaristisch verhalten und versucht, die sie Liebenden ständig im doppelten Wortsinne auszuziehen und allerlei Vorteile von ihnen zu erhaschen. Es bleibt also das Lob der wirklich, der richtig Liebenden. Schauen wir uns diese Passage doch einmal näher an:

\footnotetext{
Was würde aus dem Geliebten oder der Geliebten werden ohne jene Magie der Liebe, welche die sie Liebenden für sie erzeugen! Was würde aus diesem Stein ohne jene sanfte Brise, die ihn neuerlich zu umarmen sucht, ohne jenen Liebeswind, der die Ampeln überspringt wie ein elektrischer Strom, welcher in sich den Keim der Unsicherheit und der Hoffnung trägt! [...]

Diese Liebenden, welche ihre Zeit in Anwesenheit und in Abwesenheit verschenken, welche vor Emotion weinen, wenn sie lieben, welche beim Einschlafen noch an das letzte Wort denken, das sie von ihren Geliebten hörten, welche in einer ständigen Blase leben, welche die Probe von Weihnachten, die Probe des Sommers überstehen, welche sich dafür hergeben, Ton in den Händen der Kaprizen des Anderen zu sein, welche stumm werden bei der Anwesenheit dessen, den sie lieben: Sie verdienen die großzügigste Liebe! [...]

Die wahre Liebesgeschichte wird von jenen Liebenden in der süßen Einsamkeit ihrer Spiegel gewoben. ${ }^{3}$
}

Es ist schon erstaunlich: Das so sehr auf den spanischen Zeitgeist mit seinen Brechungen und scheinbaren Tabubrüchen berechnete Buch endet mit einer Lobpreisung der Magie der Liebe, so als ob diese Kraft - gleichsam die Liebes-

3 Rubio, Fanny: El embrujo de amar, S. 219f. 
kraft - doch niemals versiegen könne und immer wieder durchbrechen müsse. Immer wieder werde es Liebende geben, die ihrer Geliebten wegen in Tränen ausbrechen, immer wieder Autorinnen und Autoren einer wahren Liebesgeschichte, die sich letztlich immer im tragischen Modos fortspinnen werde, aber niemals zu Ende gehe. Im Grunde werden hier Versatzstücke der romantischen Liebe beschworen, jene Liebenden, die sich Hals über Kopf ohne jede Brechung oder Berechnung in die Liebe stürzen und ihre Wonnen wie ihre Schmerzen auskosten.

Es geht in dieser Passage folglich um das, was Stendhal - wie wir gesehen haben - als amour-passion bezeichnet hat, jene Magie leidenschaftlicher Liebe, die man natürlich auch mit Frankreich und mit dem Paris des 19. Jahrhunderts in Verbindung bringt. Hier nun steht am Ende die erträumte Liebesgeschichte, die historia de amor, die gleichsam gewoben wird von jenen romantischen Liebenden, die sich und ihre Liebe in der Einsamkeit ihrer Spiegel gleichsam erfinden. Diese soledad, diese Einsamkeit ist hierbei schließlich auch jene, auf die Fanny Rubio aufmerksam macht, werde doch stets nur einer der Liebenden vom Pfeil Amors getroffen, während der andere ganz entzückt ist, nun aus Liebe vergöttert zu werden. Wir haben ja schon gesehen, dass der kleine Cupido zwei Arten von Liebespfeilen in seinem Köcher hat. Auf diese Weise stehen sich somit von Beginn an stets zwei Individuen einander gegenüber, die durchaus unterschiedliche Interessen und Gefühlslagen besitzen. Und so kann die ewige Liebesgeschichte mit ihren beiden so unterschiedlichen Seiten beginnen. Einsamkeit ist dabei die eine Seite dieses Motivs.

Die andere Seite betrifft nun etwas auf den ersten Blick gänzlich Anderes. Denn am Ende steht das Spiegelmotiv, ein Motiv, auf das wir immer wieder in seinen Varianten und Variationen zurückkommen werden. Der Spiegel steht in diesem Falle nicht vorrangig für Abspiegelung wie in der Stendhal'schen Spiegelmetapher für den Roman des 19. Jahrhunderts, oder für Abbildung im Sinne einer Re-Präsentation, sondern für Selbsterkenntnis, eine Selbstschau, die letztlich stets auf die Identität des Selbst-Beschauenden zielt. Das Spiegelmotiv als Motiv der Selbstreflexivität, der Ich-Bezogenheit und des Narzissmus, aber auch der Selbstdurchdringung und Selbsterkenntnis, wird hier mit der Textgattung der Innerlichkeit verknüpft, wie wir sie freilich aus jeder besseren Schnulze, aus jeder Seifenoper, kennen. Und doch hat es dieses Motiv in sich.

Genau an diesem Punkt, an dieser wichtigen Stelle liegt eines der Probleme wie der Chancen des Buches. Denn Fanny Rubio hat in ihren Text sorgsam die unterschiedlichsten Zitate aus dem Fernsehen, vor allem aber aus dem Kino, und an erster Stelle von Boleros, Hits und Schlagern sowie anderen Liedtexten eingebaut. Sie rhythmisieren ihr Buch und bilden gleichsam den Soundtrack für eine Lektüre, in welcher die Leserschaft im Grunde immer ein wenig mitsummen 
muss. Es handelt sich um Refrains, die weit verbreitet sind und die uns vielleicht auch ein wenig an die Jugendzeit der Autorin und Professorin erinnern mögen. Auf diese zwanglose Art wird eine durchaus postmoderne Überbrückung des Gegensatzes zwischen Hochkultur und Massenkultur in Szene gesetzt, gilt die Schleifung dieses Gegensatzes doch als eine der Kernforderungen in Ästhetik und künstlerischer Praxis der Postmoderne.

Dieses Verfahren von Fanny Rubios Textgestaltung erlaubt es, neben die höchsten Höhen der Literatur den Text einer weitgehend unbekannten Schnulzensängerin zu stellen und den Verweis auf die Celestina von Fernando de Rojas mit einem Refrain aus einem Bolero geschmackvoll zu kontern. El embrujo de amar macht's möglich: Die Grenzen zwischen den einzelnen Äußerungsformen des Liebesdiskurses werden gleichsam unterlaufen und ad absurdum geführt. Was herauskommt, ist ein Liebesdiskurs, der sich auch an der Literatur, aber nicht ausschließlich an ihr orientiert. Die Liebe erscheint - ganz wie im Anfangssatz als ein Phänomen, dessen Konsequenzen unser gesamtes Leben prägen - auch wenn wir in der Liebessituation selbst die Folgen bei weitem noch nicht zu überblicken vermögen.

Man könnte folglich sehr wohl El embrujo de amar wie eine spanische Replik auf Barthes' Fragments d'un discours amoureux lesen, wobei der Band zugleich auch einen Ausblick auf die Liebe im 21. Jahrhundert ermöglicht. Denn sicherlich würden wir alle ganz gerne wissen, wie sich Schriftstellerinnen und Schriftsteller die Liebe in diesem Jahrhundert und mehr noch in der Zukunft vorstellen. Denn die Literaturen der Welt sind nicht allein Seismographen von Entwicklungen, die in Vergangenheit und Gegenwart ablaufen; den Literaturen der Welt eignet etwas Prospektives: Sie sind der Zukunft zugewandt.

So lautet das fünfzehnte und letzte Kapitel des an der Wende zum 21. Jahrhundert geschriebenen Buches auch schlicht „Amores después del 2000“. Auch wenn das Prospektive der Literatur gerade nicht in der expliziten Bezugnahme auf eine Zukunft manifest wird, möchte ich mich doch auf den Beginn dieses letzten Kapitels stürzen, um Ihnen einmal vorzuführen, wie man in Spanien am Ausgang des 20. Jahrhunderts und damit nach dem Ende der lang anhaltenden Diktatur des Generalissimus Franco über die Zukunft der Liebe im einundzwanzigsten Jahrhundert nachdachte.

Ich verzichte an dieser Stelle aus Zeitgründen auf die Darstellung der Diskussion des aus meiner Sicht wichtigen Begriffs der „sincronía“, der für das Zusammenleben der beiden Liebenden von größter Bedeutung ist. Wir finden in diesem Begriff etwas von dem vor, was Roland Barthes unter dem Konzept der Isorhythmie verhandelt hat, also jenen Bewegungen, die im selben oder gleichen Rhythmus erfolgen. Die Synchronie bei Fanny Rubio - und damit die Frage des Rhythmus - wird geradezu zum Fetisch und Glücksbringer für das Paar, denn 
diese Synergieeffekte beziehen sich auf die Liebe zwischen Liebenden, die wirklich im gleichen Rhythmus miteinander schwingen.

An dieser Stelle geht es mir aber viel mehr um einige Aspekte des Blicks auf die Liebe durch eine weibliche Autorin, die allerlei verletzte Körper in ihre Collagen einbaut. Nun also der Beginn des Kapitels über die Liebe im 21. Jahrhundert und das, was die spanische Autorin zur Liebe in der damaligen Zukunft zu sagen hatte:

In dem Maße, in welchem die Frauen neue Räume besetzen, welche traditionellerweise den Männern vorbehalten gewesen waren, und vorankommen beim Genuss ihrer ökonomischen Unabhängigkeit und anderer individueller Freiheiten, und auch in dem Maße, in welchem Männer wie Frauen sich bemüßigt fühlen, ständig ihre Haushaltsgeräte, ihre Wohnungen und ihre Partner zu wechseln, wird die Eifersucht wieder auf der Bühne erscheinen, dieser höhlenartige Wahnsinn, der bis zum Verbrechen führen kann, wenn der Stärkere dabei die Zügel aus der Hand gleiten lässt. Ich beziehe mich auf die gefühlsmäßige Eifersucht, welche die Leidensform der Eifersucht ist, obwohl die Formen beruflicher und sozialer Eifersucht auch eine verquere Haltung vieler Subjekte darstellen. [...] Die Eifersucht ist untrennbar mit der Vorstellung von Glück und den Bewegungen der Besitzergreifung bei den Paaren verbunden, und sie taucht bei der ersten Unachtsamkeit auf, auf der Bühne der allerersten Spielchen, noch bevor die ersten Liebeserklärungen kommen. [...]

Wir weibliche Liebende heterosexueller Ausrichtung müssen heute mehrere Dinge klar sehen: Das erste ist, dass wir einen und wir anderen nicht gleich sind, obwohl wir dieselben Rechte und dieselben Arbeitsplätze haben, obwohl wir an verschiedenen Stellen die Kastanien aus dem Feuer holen, obwohl wir zusammen dieselben Drinks bestellen und auch gemeinsam ins Fitnessstudio gehen. Beginnen wir mit dem Verabscheuenswürdigsten von jedem einzelnen, von jeder einzelnen: Sie spielen sich mit jeder Eroberung - gleich welcher Eroberung - in einem quantitativen Sinne auf, ich habe mehr, ich habe mehr, ich habe mehr. [...] Wir Frauen sind zurückgekehrt zum Wo und zum Wann, wir haben eine Obsession bezüglich dessen, was wir nicht sehen und dem, was abläuft, so wie wir es gerne hätten: Wo ist seine Ex-Frau, von wo aus haben sie ihn gerade angerufen, wohin geht er jetzt [...]. ${ }^{4}$

Vielleicht hätten wir mit diesem Ergebnis nicht gerechnet in einer Welt, die nicht zuletzt auch Liebe konsumiert und in der wir professionelle Partnersuche auf Knopfdruck, nicht zuletzt auch zum Zeitvertreib bestellen können. Entscheidend und gefährlich für die Liebe im längst angebrochenen Jahrhundert sind also aus Sicht einer Spanierin die Anfälle von Eifersucht und Eifersüchtigkeit, die sich ständig neue Objekte und Begründungen suchen. Interessanterweise betont Fanny Rubio in Zusammenhang damit die Veränderungen im Bereich der Emanzipation und Gleichstellung von Frauen in Spanien, beziehungsweise die Entwicklungen im Bereich der Geschlechterdifferenz, die aus ihrer Sicht signifikativ sind für eine fortschreitende Gleichberechtigung der Geschlechter. Zweifellos

4 Ebd., S. $213 \mathrm{ff}$. 
hält die spanische Schriftstellerin und Professorin diese Gleichberechtigung in vielen Bereichen auf dem Papier für fast verwirklicht, was man durchaus diskutieren kann, war diese Einschätzung doch eher der Euphorie lange nach Ende der Franco-Ära geschuldet; doch führe die fortbestehende Geschlechterdifferenz noch immer zu vielfältigen Unterschieden und Ungleichheiten in der Praxis des Lebensvollzugs - und in der Praxis heterosexueller Liebe.

Dieser Befund wird in der letzten Passage des obigen Zitats sehr deutlich. Dabei insistiert die Ich-Erzählerin - die wohl aufgrund des wenig kunstvollen Aufbaus des Bandes durchaus weitgehend mit Fanny Rubio gleichgesetzt werden kann - auf der Geschlechterdifferenz im tatsächlichen Verhalten von und zwischen Männern und Frauen. Dieses unterschiedliche Verhalten betrifft zum einen die Rechte, die sich Männer und Frauen jeweils herausnehmen und sozusagen für sich als selbstverständlich reklamieren.

Dabei erkennt sie bei den spanischen Frauen wegen einer jahrhundertelangen Unterdrückung trotz aller emanzipatorischen Entwicklungen gegenüber den Männern, den machos ibéricos, eine insgesamt noch immer stark untergeordnete Rolle und Funktion, aber auch ein problematisches Selbstbewusstsein. Zum anderen macht sie bei den Männern - und auch diesen Aspekt sollten wir nicht vergessen - ein obsessives Beharren auf der Quantität, auf der Anzahl, auf der Statistik der Liebesbeziehungen aus - und selbstverständlich auch ein dementsprechendes Geprahle. Hierzu werden im Buch selbst auch durchaus Anmerkungen gemacht, belege die Liebesaktivität in Spanien mit neunzig Liebesakten pro Jahr doch nur einen Mittelplatz zwischen den mehr als einhundertzwanzig angeblichen Geschlechtsakten US-amerikanischer Paare und den freilich mit Zurückhaltung angegebenen nur knapp vierzig sexuellen Vereinigungen japanischer Paare pro Jahr. Nun ja, da mögen auch kulturelle Faktoren mit hineinspielen. Sie wissen ja, wie Statistiken zustande kommen ...!

Überdies, so Fanny Rubio im obigen Zitat weiter, gebe es bei den Frauen die Obsession des nicht leicht Beherrschbaren, also die Abfolge von Fragen, die im Grunde nicht nach einer Statistik, sondern nach einer Geschichte fragen und ein derartiges Narrativ ständig konstruieren und kontrollieren. Interessanterweise wird gerade eine recht nahe liegende Konsequenz aus dem ständigen Nachfragen der Frauen nicht gezogen: Denn wenn die Frage nach der Statistik und der Zahl der Geschlechtsakte oder Seitensprünge gerade nicht auf narrative, sondern eher auf iterative Strukturen zielt, dann müsste ja die Frage nach dem Wo, Warum, mit Wem, Wann usw. gerade auf die ständige, unablässige Konstruktion von Liebesgeschichten (einschließlich Eifersuchtsgeschichten) zielen. Damit wäre dann vielleicht auch zu belegen, dass sich Frauen besser auf das Erzählen und das Produzieren von Liebesgeschichten verstehen und weitaus weniger leicht $\mathrm{zu}$ 
durchschauende Narrative entwickeln. Aber so weit geht die Behauptung von Fanny Rubio nicht.

Immerhin: Die Grundthese, auf die El embrujo de amar hinausläuft, ist mit anderen Worten die, dass wir in unseren Liebesbeziehungen immer weniger an bestimmte vorgestanzte Muster, und immer weniger auch an die Beibehaltung eines einmal gewählten Zustandes gebunden sind. Wir trennen uns also öfter nicht nur von den eigenen vier Wänden, in denen wir leben, sondern auch von unsren Liebespartnerinnen oder Liebespartnern, mit denen wir zusammenleben, so dass grundsätzliche Fragen des Zusammenlebens und der Konvivenz folglich wesentlich grundlegender gestellt und die Beziehungen in ihrer Gesamtheit in Frage gestellt werden als früher. Insofern scheinen sich die gesellschaftlichen und auch soziologischen Rahmenbedingungen für die Liebe im Abendland durchaus verändert zu haben.

Dies bedeutet freilich, dass es immer weniger institutionelle und auch statistische Bindungen zwischen den verschiedenen Liebespartnern gibt; und hier - so die These - taucht nun die Eifersucht auf. Denn die Eifersucht, die bekanntlich mit Eifer sucht, was Leiden schafft, ist noch immer Besitzanspruch auf den Anderen oder die Andere: Sie ist laut Rubio noch immer das Beharren eines Partners auf dem Anspruch auf sein Liebesobjekt, sein Lieblingsspielzeug, den anderen Partner.

Dabei sieht Fanny Rubio durchaus eine wohldosierte Eifersucht als eine Stütze für den Erhalt einer Paarbeziehung an, als ein gleichsam stabilisierendes Element gerade auch in der heterosexuellen Paarbeziehung. Denn die celos sentimentales, die Eifersucht der Gefühle, bilden eine erneuerte und erneuernde Beschäftigung mit dem Anderen und der Beziehung zu ihm.

Wir sollten also von Fanny Rubios Buch nicht nur bei unserem Durchgang durch die Geschichte, den wir in der Folge nun antreten wollen, die Auflösung bestimmter institutioneller Praktiken (die Unauflösbarkeit der Ehe, die rechtliche Unterwerfung der Frau usw.) und die graduelle Einführung anderer institutioneller Praktiken (etwa die Einführung der Scheidung, die leichte Trennbarkeit von Paarbeziehungen zu Singles, die wiederum neue Beziehungen eingehen usw.) sehen, sondern auch das Wiederaufleben von Besitzansprüchen dort, wo wir vielleicht so ,archaische‘ Gefühle wie die Eifersucht gar nicht vermutet hätten. Die Entwicklungen im Diskurs wie in den Praktiken der Liebe, so scheint mir, sind nicht unilinear als Entwicklung in eine einzige Richtung, sondern als viellogisch anzusehen: Die Liebe also ist, auch wenn wir sie nur im Abendland betrachten würden, komplex. Aber das wussten wir schon.

Daher verwundert es letztlich auch nicht, dass El embrujo de amar, das Buch von Fanny Rubio, auf das Lob der Liebenden in einem romantischen Sinne hinausläuft, auf eine Beschwörung der Magie der Liebe, deren Behexung 
und Verhexung ja bereits der Titel des Buches zelebriert. So entfernen wir uns zugleich von jener Liebesgymnastik, die aus wohldurchschaubaren kommerziellen Erwägungen so sehr im Mittelpunkt von El embrujo de amar steht, und die zugleich auch so opportunistisch eingebaut ist in den Liebesdiskurs des aktuellen Spanien. Wenden wir uns nach diesem Blick auf eine iberische Gegenwart, aber kaum auf eine wie auch immer genauer zu definierende Zukunft, der Historie, der Geschichte von Liebe und Lesen zu. 